\title{
Perspectiva de género en la relación entre profesionales del área de la salud con usuarios y usuarias: una revisión de la literatura
}

\author{
Gender perspective in the relationship between \\ professionals in the health area and their users: a literature review \\ Daniela Durán Rojas ${ }^{\mathrm{a}}$ \& Cory Duarte Hidalgo ${ }^{\mathrm{b}}$ \\ aUniversidad de Atacama, Chile. $\varangle$ daniela.duran@uda.cl \\ [orcid.org/0000-0002-1626-8154] \\ bUniversidad de Atacama, Chile cory.duarte@uda.cl \\ [orcid.org/0000-0002-1532-3123]
}

\section{RESUMEN}

La violencia hacia las mujeres en el ámbito sanitario se relaciona con la instauración y reproducción de modelos hegemónicos en salud que infantilizan, estigmatizan y desautorizan a las mujeres y sus saberes ancestro-tradicionales, estableciendo en el contexto clínico relaciones de poder asimétricas. El objetivo de este artículo es analizar los elementos que influyen en la relación entre profesionales del área de la salud, con sus usuarios y usuarias desde la perspectiva de género. Para ello, se efectuó una revisión sistematizada en bases de datos internacionales, utilizando los descriptores "género", "salud”, "perspectiva de género" y "biomedicina". Los resultados muestran que aspectos como género y sexo, edad, cultura y formación profesional, son determinantes en las relaciones entre el público usuario y el equipo profesional.

PALABRAS CLAVE: género, mujeres, salud, sistemas de salud.

\begin{abstract}
Violence against women in the health sector is related to the establishment and reproduction of hegemonic health models that infantilize, stigmatize and disavow women and their ancestral-traditional knowledge, establishing asymmetric power relationships in the clinical context. The aim of this article is to analyze the elements that influence the relationship between professionals in the health area with its users from gender perspective.
\end{abstract}


To this end, a systematized review of international databases was carried out, using the descriptors: "gender", "health", "gender perspective" and "biomedicine". The results show that aspects such as gender and sex, age, culture and professional training are determining factors in the relations between the user public and the professional team.

KEY WORDS: gender, women, health, health sistems.

\section{INTRODUCCIÓN}

El siguiente artículo presenta los resultados de una revisión sistematizada de la literatura efectuada con el objetivo de conocer las investigaciones científicas publicadas sobre las relaciones que establecen los y las profesionales de la salud, con sus usuarias y usuarios desde una perspectiva de género. Se utiliza esta metodología considerando la importancia y enfatizando en el carácter acumulativo del conocimiento y la ciencia, a fin de construir el estado del arte de la temática.

El modelo biomédico ha expropiado a las personas de sus cuerpos y autonomía. En el caso de las mujeres, estableció trabas en lo relativo a la salud sexual y reproductiva, imponiendo un violento control sobre los cuerpos y sus procesos, lo que implica una exclusión de la toma de decisiones sobre las propias vidas y la inclusión de los intereses de grupos privilegiados por encima de los de las mujeres. Estos elementos han sido problematizados desde los feminismos, planteando que la biomedicina, y quienes la ejercen, se erigen desde mecanismos de poder centrados en el androcentrismo y el patriarcado; en una paradoja que contradice la historia. Tradicionalmente, fueron las mujeres quienes ejercieron labores de cuidado y sanación en las comunidades y hogares, pero fueron exiliadas en el nuevo modelo de comprender la medicina, despojándole de la tradición ancestral para entregar dicho poder al personal médico (Obach \& Sadler, 2009).

Quienes son profesionales del área de la salud, en particular matronas y obstetras, que centran su quehacer en el trabajo directo con niñas, adolescentes y mujeres, cumplen un rol fundamental a la hora de construir, apropiar y expropiar autonomías y libertades respecto a los cuerpos de las mujeres. Entendiendo todo ello, desde las distintas perspectivas en las que se posicionan ante el ejercicio profesional, el equipo sanitario puede reproducir y perpetuar patrones violentos impuestos a través del predominio de un modelo biomédico que contiene en sí mismo lógicas patriarcales evidentes. El modelo biomédico, tanto en su origen como en sus fundamentos teóricos y políticos, y hasta en las prácticas cotidianas en los servicios de salud, evidencia tratos asimétricos en el contexto clínico entre usuarias y el equipo de profesionales que las atienden (Riquelme, 2011).

El modelo basado en la fisiopatología genera un conocimiento fragmentado, de temporalidad lineal, en el que reina la lógica por encima de las emociones. Un esquema en el que espíritu y materia se encuentran separados, y en el que prevalece la ciencia y la técnica. 
Esos conocimientos, y la estructura que se ha construido en torno a su prestigio, genera relaciones de dominio y obediencia entre profesionales y personas usuarias. En la relación entre médico y paciente es observable dicha asimetría: médico es quien sabe y paciente es quien ignora, en una relación en la que quien ocupa el lugar de privilegio lo hace asociado a un contexto patriarcal y capitalista que sustenta su dominio (Linardelli, 2015; Zacher, 2015; Sibrian, 2016). En ocasiones, esto puede generar situaciones de pérdida de autonomía que son profundizadas al cruzar otras variables de opresión como género o raza. Para quienes investigan, el interés particular fue observar las formas en que se daban estas relaciones cuando se considera la perspectiva de género, a fin de seguir profundizando en la temática.

\section{MÉTODO}

El estudio consideró una revisión sistematizada de la literatura realizada entre los meses de abril y mayo del año 2018, para lo cual se analizaron publicaciones de revistas científicas contenidas en las bases de datos internacionales, aplicando un límite temporal desde el 2013 al 2018, con la finalidad de identificar artículos actualizados.

Una revisión sistematizada es el reconocimiento de las investigaciones científicas y toda la información disponible sobre un tema en específico, de distintas áreas del conocimiento. Se centra en el análisis y la exploración de lo que se ha investigado. Busca encontrar vacíos en el conocimiento y espacios para indagar, además de identificar áreas y corrientes principales del saber en la temática escogida y demostrar que quien investiga se ha empapado de los aprendizajes obtenidos de otros investigadores, haciendo referencia a la importancia del carácter acumulativo de la ciencia. Tiene como finalidad construir el estado de la cuestión, por medio de estrategias metodológicas que se van delimitando a lo largo del proceso y etapas bien definidas: búsqueda, evaluación, análisis y síntesis (Codina, 2018).

La búsqueda de las investigaciones incluidas en esta revisión se realizó en las bases de datos electrónicas Dialnet, Scopus y Web of Science (WOS), a través de palabras clave contenidas en los artículos, a fin de identificar aquellos publicados recientemente en relación con la temática del género en la salud y cómo esto influye en las relaciones que se dan a nivel sanitario.

La selección de las investigaciones que componen el corpus de esta revisión se realizó en función de criterios como el idioma del artículo, inglés y español; tipo de investigación, esto es, artículos de revisión o estudios secundarios y artículos científicos de investigación primaria cualitativa, cuantitativa o mixtas.

Se utilizaron los descriptores: "género”, “salud”, "perspectiva de género”y "biomedicina”, contenidos en la Lista de descriptores del Consejo Latinoamericano de Ciencias Sociales (CLACSO), tanto en inglés como en español. Se dejaron de lado otras palabras relacionadas como mujer/mujeres o medicina/médico, por lo amplio de los conceptos y su aplicabilidad a distintos contextos, lo que escapa del objetivo de esta revisión. 
Del total de 30 investigaciones pertinentes a la temática encontradas, se han seleccionado 22 documentos de interés; 11 artículos científicos de carácter primario y 11 artículos científicos de investigaciones secundarias.

Se excluyeron de esta revisión los trabajos que, pese a cumplir con los requisitos previos, estaban centrados en lo estrictamente epidemiológico, los casos sociales o de salud muy particulares, las enfocadas en el género como barrera de acceso a servicios de salud específicos y los trabajos relacionados con usuarios transgénero, transexuales o intersexuales.

\section{RESULTADOS}

En este apartado constan los resultados obtenidos tras la revisión de los artículos científicos seleccionados, los que son presentados en distintas categorías, estas son: hegemonía biomédica; relación personal/población usuaria; atención del parto y violencia obstétrica; género, diferencias y accesos; niñas, jóvenes y mujeres; formación; y, por último, estereotipos.

\section{Hegemonía biomédica}

El modelo biomédico que hoy rige a los sistemas de salud tiene fundamentos científicos y clínicos que se han extendido y profundizado con el avance de las tecnologías, tratamientos y la construcción de conocimiento. Este modelo está basado en las lógicas de pensamiento racionalista cartesiano y en la física newtoniana en las cuales los cuerpos son considerados máquinas biológicas dualistas, compuestas por partes que funcionan con base a leyes universales (Baeta, 2015). Desde entonces, la aceptación del modelo se extendió y contribuyó a la erradicación de enfermedades infectocontagiosas, disminuyendo las tasas de mortalidad a nivel mundial, lo que impactó con fuerza en el desarrollo, investigación e implementación de múltiples avances tecnológicos para prevenir y sanar enfermedades, entre otros aportes a la salud de la población humana (Baeta, 2015).

En concordancia con el contexto social y político actual, es posible visualizar que el modelo biomédico ha instaurado estructuras hegemónicas que delimitan los procesos de salud-enfermedad centrados en la patología y en el tratamiento de ésta a través de distintos métodos aprobados por la comunidad médica. La salud se convierte en un territorio de dominio de la medicina donde otros saberes y conocimientos no tienen cabida, favoreciendo una relación asimétrica entre quienes ostentan el poder: profesionales de la medicina por sobre la población usuaria, soslayando la autonomía, la autodeterminación, los saberes populares y los conocimientos ancestrales de los pueblos y las personas (Linardelli, 2015; Zacher, 2015). La historia, la cultura, el saber popular, las subjetividades

e intersubjetividades de la población y tantos otros elementos contextuales intrínsecos de las personas y los pueblos, quedan excluidos en esta concepción de la realidad, 
puesto que este paradigma se basa en lógicas androcentristas, reduccionistas, curativas, segregacionistas, patriarcales y elitistas que han legado la construcción de relaciones asimétricas y violentas, además de la medicalización de procesos naturales del ámbito físico, biológico, social y mental para ejercer poder y control (Urrea, 2014; Linardelli, 2015; Zacher, 2015).

El modelo biomédico se centra en los aspectos biologicistas y cientificistas, tanto en la clínica como en la investigación, así deja de lado otros factores que también participan en los procesos de salud-enfermedad, como son los aspectos socioeconómicos, culturales, de género, de raza, etc. a los que se denominan determinantes sociales de la salud (Urrea, 2014; Panisello \& Pastor, 2015; Escobar-Ballesta, García-Ramírez, Albar-Marín \& Paloma, 2017).

Las determinantes sociales están en el tapete nuevamente, luego de que el sistema construyó una realidad a partir de la que se ha producido una "exclusión de saberes y conocimientos alternativos, que se caracterizan por utilizar lógicas explicativas menos restrictivas y más complejas, como también por considerar la salud como resultado de la interacción de múltiples determinaciones sociales, políticas, culturales y económicas" (Linardelli, 2015, p. 217).

En algunas investigaciones, se evidencia que hay disciplinas que tienen o ejercen mayor poder y control sobre las personas usuarias y también sobre otras disciplinas del ámbito sanitario, de forma principal en aquellas que se dedican a la atención de las mujeres (Camacaro, et al. 2015; Cassiano et al. 2016; Linardelli, 2015; Pozzio, 2014 \& Urrea, 2014). Este sistema de dominación tiene sentido si consideramos que la medicina es una rama del conocimiento que está construida por hombres y para hombres. Ejemplo de esto, son los discursos recopilados por Pozzio (2014) de profesionales del área de la salud sexual y reproductiva de las mujeres, en los que se señalaba el predominio de "actitudes que son profundamente misóginas y que están fundadas estructuralmente en el ejercicio de la especialidad y en la invención de la especialidad, en términos que es una especialidad básicamente manejada e inventada por los varones" (p. 333).

Distintos estudios, en diversas situaciones y contextos (Terán, Castellanos, González \& Ramos, 2013; Urrea, 2014; Gomes \& Couto, 2014; Linardelli, 2015; Zacher, 2015; Camacaro et al. 2015; Tasa-Vinyals, Giral \& Raich, 2015; Valdés, Palavecino, Pantoja, Ortiz \& Binfa, 2016; Triuzzi, 2017; Rangel-Flores \& Martínez-Ledezma, 2017) coinciden en señalar que, para las mujeres, el sistema biomédico es violento y coercitivo, sobre todo en cuanto a la salud sexual y reproductiva. El parto, la menstruación y el control natal, que antes estaban en manos de las parteras, comadronas u otras mujeres que atendían con base en sus conocimientos ancestrales, han sido medicalizados. La medicalización impuesta por este sistema, en una sociedad capitalista, se instaura como un elemento de control económico y social, ya que la salud es entendida como falta de enfermedades o la curación de éstas, y la sitúa como un bien de consumo al que no todas las personas pueden acceder. 
La discriminación a las mujeres en salud tiene relación con los factores estructurales ya enunciados, los que también se expresan en la invisibilización de sus cuerpos y la consideración de lo masculino como norma, de forma que, existe una "aplicación mecánica y directa de los resultados obtenidos en varones a ellas” (Linardelli, 2015, p. 208), y que se considera el cuerpo masculino como modelo o ideal de ser humano y al femenino como una reproducción de este (Linardelli, 2015; Panisello \& Pastor, 2015; Tasa-Vinyals et al. 2015).

\section{Relación personal/población usuaria}

En cuanto a las relaciones entre usuarias y profesionales, Valdés et al. (2016) sostienen en su investigación que las experiencias de las mujeres mejoran cuando el equipo médico las percibe como personas, sujetas activas de derechos, autónomas y con capacidad para participar y decidir en sus cuerpos y procesos corporales. El sistema hegemónico biomédico dificulta este tipo de relaciones porque, según Pitter (2016), existen elementos como el tiempo, el tipo y la calidad de atención que se espera brinde el equipo profesional para ser efectivos y eficaces, que se convierten en barreras para el establecimiento de relaciones más cercanas.

Por otra parte, y desde la realidad rural en México, en la investigación de Villaseñor, Laureano, Mejía, Valadez, Márquez y González (2014), las parteras o matronas que atienden a la población residente en este contexto no están insertas en un sistema médico cerrado y/o establecido. Algunas de ellas no cuentan con formación profesional o capacitación formal y se centran más bien en sus experiencias y en los conocimientos adquiridos por medio de la tradición y el saber popular. Estas matronas asumen un rol activo en la detección de la violencia y en la atención previa, durante y posterior al parto, debido a que las mujeres en dicha etapa se encuentran en una situación de alta vulnerabilidad.

El acompañamiento de las matronas en las condiciones descritas, resulta ser fundamental en sociedades profundamente machistas en las que muchas de estas mujeres, dadas sus particulares circunstancias, son más propensas a ser víctimas de violencia física, sexual, psicológica o emocional, por abandono y/o por omisión de sus derechos (Villaseñor et al. 2014) al interior de sus familias y en cualquier aspecto de la vida cotidiana. Es interesante lo señalado aquí, puesto que se plantea que los equipos médicos deberían estar capacitados para detectar la violencia intrafamiliar y contar con protocolos establecidos, conocidos y aceptados para intervenir de forma respetuosa y efectiva ante estas situaciones. El rol que cumple la partera, más allá de su rol de experta, es de vital importancia a la hora de mantener relaciones de carácter simétricas, en las que la ética del cuidado, el acompañamiento y colaboración entre congéneres sean elementos basales, así se incentiva la autonomía del cuerpo y las decisiones de las mujeres embarazadas o en labores de parto (Valdés et al. 2016; Villaseñor et al. 2014). 
PERSPECTIVA DE GÉNERO EN LA RELACIÓN ENTRE PROFESIONALES DEL ÁREA DE LA SALUD

Atención del parto y violencia obstétrica

A partir de la instauración de la matronería, como un proceso biomédico y como profesión, los nacimientos y partos pasan del ámbito domiciliario y de ser propiedad de las mujeres a ser parte de un sistema hegemónico que impone paradigmas, reglas, métodos y formas de ver la vida, la salud y la enfermedad (Villaseñor et al. 2014; Zacher, 2015; Cassiano et al. 2016; Valdés et al. 2016; Triuzzi, 2017). La evolución de la matronería desde lo intrínsecamente mecanicista y biomédico hasta la actividad profesional más integral de hoy en día, es significativa en cuanto a las relaciones que se establecen con las personas, dado que las acciones desarrolladas pueden significar experiencias positivas o negativas para las mujeres que reciben atención, dependiendo no solo de las competencias profesionales del matrón o matrona sino también de cómo se relacione con las mujeres que atiende, el sistema en el que se desenvuelve y el equipo técnico y profesional que le acompaña.

Hoy, la violencia contra las mujeres está presente en todo ámbito de la vida de las personas. Estas violencias constituyen una vulneración directa a los derechos humanos al dificultar el acceso al íntegro desarrollo social e individual (Bello-Urrego, 2013; Terán et al. 2013; Villaseñor et al. 2014; Gómez-Fernández, Isidro-Albadalejo, García-del Arco \& Molina-Ordóñez, 2017). Una mujer o una niña, como persona y como usuaria de un sistema de salud, no solo necesita profesionales capacitados, sino que también requiere personas capaces de entregar compañía y empatía, que la hagan sentir segura y satisfecha con el servicio que recibe, autónoma, educada y empoderada para poder decidir sobre su propio cuerpo.

Los partos y las atenciones relativas a éstos, es decir, controles previos, trabajo de parto, postparto y puerperio, son abordados, generalmente, por profesionales de la matronería, ya que son estas las personas capacitadas y formadas para atender tanto a las mujeres como a sus recién nacidos (Valdés et al. 2016). La medicalización de los nacimientos, tras el ya mencionado cambio del parto en casa a la institucionalización de este proceso (Villaseñor et al. 2014; Casiano et al. 2016; Valdés et al. 2016; Zacher, 2015; Triuzzi, 2017), ha implicado considerables modificaciones en la salud materna-infantil y en la relación entre usuarias y el equipo profesional que brinda atención, a lo que se suma el avance en el uso de analgesia, antibióticos y mejora en los procedimientos, lo que desembocó en una reducción de la mortalidad materno-fetal (Casiano et al. 2016).

Pese a los avances en la medicina y tecnología biomédica, la noción de violencia obstétrica permanece. La violencia obstétrica se define como "la apropiación del cuerpo y procesos reproductivos de las mujeres por personal de salud, que se expresa en un trato deshumanizador, en un abuso de medicalización y patologización de los procesos naturales, que traigan pérdida de autonomía y capacidad de decidir libremente sobre sus cuerpos y sexualidad" (Terán et al. 2013, p. 174). Esto se expresa a través de diferentes tipos de 
violencias, las que configuran mecanismos de control social por parte del sistema biomédico androcéntrico y patriarcal (Pozzio, 2014; Urrea, 2014; Linardelli, 2015). A continuación, se detallan algunas manifestaciones de la violencia obstétrica:

- Violencia institucional: acciones, decisiones u omisiones que realizan las personas que desempeñan labores y entregan atención a otras en el sistema público, que atentan contra los derechos y la dignidad de las mujeres (Terán et al. 2013). Se asocia este tipo de violencia a la precarización de los sistemas biomédicos formales, a la falta de ética que existe entre el grupo de profesionales y a la jerarquización de las relaciones (Cassiano et al. 2016).

- Violencia simbólica: "son mensajes, valores, iconos, signos que transmiten y reproducen relaciones de dominación, desigualdad y discriminación en las relaciones sociales que se establecen entre las personas y naturalizan la subordinación de la mujer” (Terán et al. 2013, p. 174).

- Violencia obstétrica: se evidencia en prácticas de rutina normalizadas, como la medicalización para acelerar el parto, realización de la episiotomía, la autoestimulación de los pezones para aumentar la intensidad de contracciones uterinas, revisiones uterinas para acelerar la expulsión de la placenta (Camacaro et al. 2015), esterilización forzada o privación de la capacidad reproductiva de la mujer, sin previo aviso o autorización (Terán et al. 2013), entre tantas otras existentes.

\section{Género, diferencias y accesos}

Cuando se utiliza el concepto género de forma frecuente se confunde con el concepto sexo. El género es una "construcción social de lo que se considera ser mujer u hombre (...) Como construcción social dinámica, el concepto se forma, reforma y evoluciona constantemente basado en el poder y las normas socioculturales vigentes sobre hombres y mujeres" (Linardelli, 2015 p. 15). Entonces, cuando se habla de género, no solo se habla de mujeres, sino que se apela a las relaciones que socialmente se han construido con base en las diferencias sexuales.

En una investigación realizada en el contexto de los sistemas de salud brasileños se señala que son incuestionables las diferencias existentes entre hombres y mujeres y que, en lo sanitario, los estudios específicos relativos a hombres son realizados recién a partir de mediados de los años noventa del siglo pasado; al mismo tiempo, se entrega poca relevancia al contexto sociocultural en el que se construyen las relaciones de género. Es aquí donde se destaca el carácter relacional de la perspectiva de género, la cual podría explicar las construcciones sociales jerarquizadas que se han instaurado a partir de estas diferencia, de esta forma entonces, es ineludible que existe un "origen político en el concepto de género" (Gomes \& Couto, 2014, p. 354). 
La perspectiva de género, según Fanny Urrea, “aporta a la bioética el análisis y comprensión del conocimiento sobre el manejo desigual, homogéneo y asociado a la «naturaleza», que han tenido las mujeres por el hecho de ser corporalmente diferentes" (Gomes \& Couto, 2014, pp. 229-230). En esta relación con el cuerpo, el género y la bioética encuentran un punto de convergencia. Desde el primero, se entienden las corporalidades como múltiples diversidades que se encuentran sometidas a distintas formas de control y poder, mientras que la bioética plantea que el cuerpo se somete a los distintos avances tecnológicos, biomédicos y científicos. Por el contrario, desde la bioética es posible destacar uno de sus principios fundamentales, la autonomía y la justicia (Urrea, 2014), ya que se entiende que las mujeres se encuentran en una situación de desequilibrio y desigualdad en las relaciones y en el acceso.

Concebir las prácticas de atención en el sistema médico formal y la investigación en este ámbito, desde una perspectiva de género, permitiría un mejor diseño y acceso a los programas de prevención y promoción, ampliando a toda la población los servicios que durante años estuvieron centrados en la salud materna y en la infantil. Proponen TasaVinyals et al. (2015) que, mientras el modelo predominante no se interese en hacer partícipe a la perspectiva de género en su forma de construir realidades, se favorece la perpetuación de las creencias, estereotipos, sesgos y actitudes sexistas por parte de las personas cuyo quehacer profesional se vincula al ámbito sanitario, por tanto es de suma importancia educar y formar tanto a las nuevas generaciones de profesionales como a las que ya se encuentran ejerciendo, en temáticas de género y equidad.

A la hora de hablar de inequidades es necesario comprender que el género no es la única limitante que impone el sistema médico formal. Linardelli (2015) hace referencia a la interseccionalidad, en donde se conjugan distintos factores que influyen en el acceso y las relaciones de poder que existen dentro de un contexto sociocultural, económico y biomédico.

La interseccionalidad hace referencia a las formas en que distintos aspectos de la vida se intersectan y configuran a las personas y sus identidades como tales. Las intersecciones se relacionan con desigualdades asociadas a la raza, la clase, el género, la edad, la orientación sexual u otros que les sitúa en alguna situación particular, de privilegio u opresión. La comprensión de la realidad a través de la interseccionalidad permite develar la manera en que estas características, individuales o comunitarias al encontrarse, implican diferentes tipos de discriminaciones, vulneraciones y limitaciones a la hora de ejercer sus derechos. Los sistemas crean y recrean desigualdades teniendo en cuenta los aspectos sociales, políticos, culturales, personales e históricos de cada una de ellas. En el sistema biomédico se acentúan estos aspectos, a través de la medicalización, falta de autonomía en los procesos de saludenfermedad y otras formas de violencia institucionalizada, las que se presentan como forma de control social hacia quienes no se enmarcan en el prototipo androcéntrico y hegemónico de quién ejerce el poder (Gomes \& Couto, 2014; Linardelli, 2015; Tasa-Vinyals et al. 2015; Pitter, 2016; Cassiano et al. 2016; Crockett \& Cooper, 2016; Zacher, 2015). 


\section{Niñas, jóvenes y mujeres}

Teniendo en consideración que la relación entre profesional y usuario o usuaria no se da solo en contextos de parto o atención gineco-obstetra, se incluye el artículo de Tajer et al. (2015) en el cual se vislumbra el enfoque de género desde el acceso y atención de salud para niños y niñas, así como las decisiones y construcciones que realiza el equipo profesional en torno a ello. Postula que los idearios de las personas profesionales que trabajan en servicios públicos y privados de salud con población infantil consideran que niños y niñas no tienen mayores diferencias en el acceso a la salud durante la primera infancia, pero estas diferencias existen y se comienzan a hacer notorias al crecer y acercarse a la adolescencia, lo cual tiene directa relación con la crianza y la sociedad actual. En los resultados del estudio realizado en Argentina, aparecen entre las principales diferencias de género las pautas de juego y recreación, así como la forma en que niños y niñas son socializados; asimismo, se observan diferencias en la percepción que se tiene sobre los accidentes de hombres y mujeres, y en las temáticas asociadas a nutrición y alimentación, las que se establecen en función de estereotipos y roles de género existentes en la sociedad patriarcal, que normaliza conductas y actitudes como propias de los géneros.

El equipo de profesionales de la salud poco releva los factores sociales, culturales y económicos que influyen en el estado de salud de niños, niñas y sus familias en general. La conciencia sobre estas diferencias en los distintos contextos socioeconómicos, sumada a la perspectiva de género, sería utilidad a la hora de educar a las familias y entregar un mayor bienestar a la población infantil. Esto permitiría trabajar en la identificación y atención de los estereotipos y sesgos de género que presentan los equipos profesionales en sus acciones y discursos, así se intenta evitar la naturalización y normalización de situaciones y condiciones asociadas a construcciones genéricas (Tajer et al. 2015).

Crocket y Cooper (2016) proponen que la inclusión del género de forma transversal en los ámbitos sanitarios a lo largo de todo el ciclo vital, permite mejorar la calidad del servicio, planes y acciones con miras a una salud integral y equitativa más allá de lo relativo a la planificación familiar o a la reproducción como tal, para extender las atenciones clínicas y la investigación no solo al grupo de mujeres en edad fértil, sino también a otros grupos de la población femenina, como lo son las niñas y las mujeres ancianas. El modelo biomédico centra su atención en las madres, cuidadoras y reproductoras, excluyendo a aquellas mujeres que requieren cuidados y atenciones diferenciadas.

El poder y la relación asimétrica que existe entre las familias y las personas que se desempeñan en el sistema médico tradicional, contribuyen a la mantención y construcción de idearios estereotipados de género, socioeconómicos y de crianza, ya que estas personas participan de distintas formas en los procesos de crecimiento y de salud-enfermedad, centrado en los primeros años de vida de la población usuaria. Del texto de Tajer et al. (2015) se desprende que la integralidad y la flexibilidad de la atención que deben brindar los distintos grupos que interactúan en el sector salud son claves a la hora de tener poblaciones 
infantiles saludables, ya que, además, están llamados a detectar posibles episodios de malos tratos u omisiones por parte de las familias. La interdisciplinariedad y el enfoque de género, entonces, son claves a la hora de entregar un servicio de calidad y así evitar sesgos como "la entrega de una atención igual para ambos sexos cuando las necesidades de género no son iguales, en la entrega de atención diferente cuando las necesidades no son diferentes y en un trato que refuerza los estereotipos de género" (Tajer et al. 2015, p. 104).

\section{Formación}

La formación profesional del equipo que se desempeña en el ámbito clínico es fundamental y ésta debe considerar los aspectos técnicos, éticos y relacionales (Valdés et al. 2015) para entregar una atención oportuna, eficaz e integral a su población usuaria. La capacitación, por otra parte, ha de ser constante y extenderse más allá de la etapa de formación académica o universitaria, en un proceso que continúa en la vida laboral. Este esencial elemento dista de estar presente en la realidad vislumbrada en el contexto actual, ya que la formación profesional en las distintas áreas de la preparación biomédica "se distancia cada vez más de la integralidad, para fraccionar el cuerpo y centrarse en el manejo aislado" (Urrea, 2014, p. 227).

En lo relativo a la atención femenina, el equipo profesional de la matronería y obstetricia ve disminuida su capacidad de intervención y de relación efectiva-afectiva con sus usuarias, debido a las pocas instancias de preparación en cuanto a temáticas de género y violencia de género; "la partera es el personaje que recibe menos o nula sensibilización, capacitación y actualización sobre violencia contra la mujer, pese a su influencia y posicionamiento social para vivir de manera más cercana la violencia experimentada por mujeres embarazadas" (Villaseñor et al. 2014, p. 11). Las prácticas de violencia institucionalizada, discriminación y estereotipos contra las mujeres y contra quienes forman los grupos disidentes y excluidos en los servicios de salud, podrían ser trabajadas en la formación y capacitación del personal de salud, las que deben estar orientadas a implementar el enfoque de género a fin de entregar una atención más justa, equitativa, inclusiva e integral (Panisello \& Pastor, 2015; Tajer et al. 2015; Tasa-Vinyals et al. 2015; Pitter, 2016; Rangel-Flores \& Martínez-Ledezma, 2017). Un equipo médico que tiene las herramientas y competencias para visualizar y actuar en una realidad desde la perspectiva de género como paradigma, es capaz de velar por los derechos de las personas y ser significativo en procesos de empoderamiento y educación popular, por la relación directa y constante que se tiene con la población.

\section{Estereotipos}

Respecto a los estereotipos de género que se perpetúan en el ámbito sanitario, es necesario mencionar que éstos se enmarcan en patrones que se repiten en los discursos, las acciones y decisiones que toma un equipo médico y cada profesional que lo conforma. 
En cuanto a la salud masculina, estos estereotipos según Gomes y Couto (2014) se dan con relación a la negatividad ante la incorporación de los hombres, como usuarios, en las atenciones de salud de rutina, porque históricamente se han postergado a favor de la atención de las mujeres, lo que impide un cuidado integral de la población. El modelo genera estereotipos en lo femenino y en lo masculino, y se espera de las mujeres que se cuiden a sí mismas y que, además, sean cuidadoras de otras personas (Urrea, 2014; Gomes \& Couto, 2014; Triuzzi, 2017).

Estas normas sociales establecidas en cuanto al género en las mujeres se dan en distintas áreas. En cuanto a la salud mental, Linardelli sostiene que las labores de crianza, cuidado y roles similares asociados a las mujeres contribuyen a crear procesos de enfermedades mentales, vinculadas a la vulnerabilidad que se asocia al género femenino (Linardelli, 2015, p. 210).

En tanto, en otros ámbitos de la salud y la vida en sociedad "el cuerpo de las mujeres es un estereotipo «construido» socialmente, centrado en la maternidad” (Urrea, 2014, p. 194); gran parte de los estudios e investigaciones realizados en los últimos años en cuanto a mujeres y salud refieren principalmente a los procesos relativos a la atención del parto y están de acuerdo en la existencia de un encasillamiento y estereotipo femenino vinculado a la maternidad y a los cuidados de la progenie (Urrea, 2014; Panisello \& Pastor, 2015; Tajer et al., 2015;Crockett \& Cooper, 2016; Triuzzi, 2017), transformándole "en un lugar de subordinación para las mujeres” (Triuzzi, 2017, p. 101), así se invisibiliza a aquellas otras mujeres que se encuentran fuera de la edad reproductiva dado que en la salud femenina se enfatiza la maternidad (Crockett \& Cooper, 2016).

El estereotipo y la dualidad casi inseparable de mujer/madre limitan la comprensión de que distintas mujeres tienen distintas identidades, que mutan y se van construyendo a través de las interrelaciones y los devenires del contexto. Las mujeres no constituyen una categoría homogénea, invariante ni estática (Urrea, 2014).

\section{CONCLUSIONES}

El objetivo de esta investigación fue proporcionar una aproximación a los aspectos relativos al género que influyen en la relación que se da en los contextos de atención clínica entre el equipo profesional, principalmente el vinculado a la atención a las mujeres, y la población usuaria, a través de la revisión y análisis de la literatura científica reciente disponible.

Si bien el modelo biomédico hegemónico se ha instalado con fuerza y buenos resultados en el ámbito sanitario, ha excluido aspectos sociales, culturales, económicos y ambientales que son trascendentales para la calidad de vida y dignidad de las personas y los grupos sociales. Este paradigma ha expropiado a las personas de sus cuerpos y autonomía, principalmente en cuanto a las mujeres y en lo relativo a la salud sexual y reproductiva, donde se ha ejercido 
violentamente control de los procesos netamente femeninos, en los que ellas han quedado fuera de la toma de decisiones y éstas se han determinado a partir de los intereses de grupos privilegiados, que ostentan el poder y que perpetúan ideologías, metodologías y perspectivas que despersonalizan a todos quienes se involucran en los procesos de salud y enfermedad, estableciendo lógicas de subordinación femenina a través de estereotipos y acciones u omisiones que atentan contra la dignidad y autonomía de las mujeres.

La violencia obstétrica es una forma específica de violentar a las mujeres, de forma física, psicológica, emocional, etc., la que se da en los contextos de atenciones médicas relativas a la salud sexual y reproductiva, como consultas o controles de rutina o de manera más explícita y violenta en los partos y postpartos, donde se elimina la autonomía y la autodeterminación de las mujeres y existe una apropiación de sus cuerpos y procesos naturales, por medio de la implantación y mantenimiento de modelos hegemónicos, impersonalizados y violentos que atentan contra la libertad personal y ciudadana.

La incipiente y tardía formación de personal especialista en la atención de mujeres desde una perspectiva de género acentúa esta situación de vulneración sistemática de los derechos de las mujeres, que no solo se da a nivel sanitario, sino que abarca las dimensiones sociales, culturales, familiares, productivas, etc. La educación, en todos sus niveles, ha de ser inclusiva, no sexista y feminista a fin de generar personas y profesionales integrales, que crean en la equidad y la justicia social.

\section{REFERENCIAS}

Baeta, M. (2015). Cultura y modelo biomédico: reflexiones en el proceso de salud enfermedad. Comunidad y Salud, 13(2), 81-84.

Bello-Urrego, A. (2013). Sex/gender, violence and human rights: Conceptual perspectives for approaching gender-based violence against women from the health sector. Revista Colombiana de Psiquiatria, 42(1), 108-119.

Camacaro, M., Ramírez, M., Lanza, L., \& Herrera, M. (2015). Conductas de rutina en la atención al parto constitutivas de violencia obstétrica. Utopía y Praxis Latinoamericana, 20(68), 113-120.

Cassiano, A., dos Santos, M., dos Santos, F., de Holanda, C., Leite, J., de Maranhão, T., \& Enders, B. (2016). Expresiones de violencia institucionalizada en el parto: una revisión integradora. Enfermería Global, 15(44), 452-464.

Crockett, C., \& Cooper, B. (2016). Gender norms as health harms: reclaiming a life course perspective on sexual and reproductive health and rights, Reproductive Health Matters, 24(48), 6-13.

Codina, L. (2018). Revisiones bibliográficas sistematizadas: Procedimientos generales y Framework para Ciencias Humanas y Sociales. Barcelona: Máster Universitario en Comunicación Social. Departamento de Comunicación. Universitat Pompeu Fabra. 
Recuperado de https://repositori.upf.edu/bitstream/handle/10230/34497/Codina_ revisiones.pdf

Escobar-Ballesta M., García-Ramírez, M., Albar-Marín, M. \& Paloma, V. (2017). Salud sexual y reproductiva en mujeres gitanas: el programa de planificación familiar del Polígono Sur. Gaceta Sanitaria, 33(1), 222-228.

Gomes, R., \& Couto, M. (2014). Relaciones entre profesionales de la salud y usuarios/as desde la perspectiva de género. Salud colectiva, 10(3), 353-363.

Gómez-Fernández, M., Isidro-Albadalejo, M., García-del Arco, M., \& Molina-Ordoñez, R. (2017). Aspectos bioéticos de la relación asistencial de las matronas con las mujeres embarazadas en contexto de violencia de género. MUSAS. Revista de Investigación en Mujer, Salud y Sociedad, 2(2), 40-55.

Linardelli, F. (2015). Salud mental y género. Diálogos y contrapuntos entre biomedicina, feminismos e interseccionalidad. Millcayac - Revista Digital de Ciencias Sociales, 2, 199224.

Obach, A., \& Sadler, M. (2009). Cuerpo femenino, medicina y poder: reflexiones en torno a las disrupciones en la atención de salud reproductiva. En Red Chilena contra la violencia doméstica y sexual (Ed.), Nación Golpeadora; Manifestaciones y Latencias de la Violencia Machista (pp. 80-90).

Panisello, M., \& Pastor, I. (2015). Salud con igualdad: una propuesta para la incorporación de la perspectiva de género en los sistemas sanitarios. Ciência \& Saúde Coletiva, 20(5), 1555-1563.

Pitter, C. (2016). Midwives' knowledge and attitudes when encountering Gender-Based Violence in their practice at a maternity-hospital in Kingston, Jamaica. International Journal of Qualitative Studies on Health and Well-being, 11(1).

Pozzio, M. (2014). El hecho de que sean más mujeres, no garantiza nada: feminización y experiencias de las mujeres en la ginecobstetricia en México. Salud colectiva, 10(3), 325-337.

Rangel-Flores, Y., \& Martínez-Ledezma, A. (2017). Ausencia de percepción de violencia obstétrica en mujeres indígenas del centro norte de México. Revista Conamed, 22(4), 166-169.

Ríos, L. (2011). Una reflexión acerca del Modelo de Investigación Biomédica. Salud Uninorte, $27(2), 289-297$.

Riquelme, G. (2011). Relación matrona-usuaria en atención primaria de salud. Horiz Enferm, 22 (1), 55-63.

Sibrian, N. (2016). De máquina a proyecto: el cuerpo en el nuevo espíritu del capitalismo. Rev. Reflexiones, 95(1), 143-155.

Tajer, D., Reid, G., Gaba, M., Cuadra, M., Lo Russo, A., Salvo, I., \& Solis, M. (2015). Equidad de género en la atención de la salud en la infancia. Psicoperspectivas, 14(1), 103-113.

Tasa-Vinyals, E., Giral, M., \& Raich, R. (2015). Sesgo de género en medicina: concepto y estado 
de la cuestión. Cuadernos de medicina psicosomática y psiquiatria de enlace, 113, 14-25. Terán, P., Castellanos, C., González, M., \& Ramos, D. (2013). Violencia obstétrica: percepción de las usuarias. Revista de Obstetricia y Ginecología de Venezuela, 73(3), 171-180.

Triuzzi, S. (2017). ¿Es feminista parir en casa? Dilemas y contradicciones en la relación entre feminismo y parto en casa. Revista Andaluza de Antropología, 13, 85-111.

Urrea, F. (2014). Respeto a la autonomía de mujeres gestantes en salud: diálogo bioéticagénero. Revista Colombiana de Bioética, 9, 193-236.

Valdés C., Palavecino N., Pantoja L., Ortiz J., \& Binfa L. (2016). Satisfacción de la mujer respecto al rol de la matrona/matrón en la atención del parto, en el contexto del modelo de atención personalizada en Chile. Matronas Profesión, 17(2), 62-69.

Villaseñor M., Laureano J., Mejía, M., Valadez, I., Márquez, J., \& González J. (2014). Mujeres violentadas durante el embarazo y el parto: experiencias de parteras en Jalisco, México. Revista Facultad Nacional de Salud Pública, 32(2), 9-16.

Zacher, L. (2015) Obstetrics in a Time of Violence: Mexican Midwives Critique Routine Hospital Practices. Medical Anthropology Quarterly, 29(4), 437-454. 